\title{
Determination of Band Structures of InN/GaN Interfaces by Synchrotron Radiation Hard X-ray Photoemission Spectroscopy*
}

\author{
Yasushi Toyoshima \\ Department of Applied Chemistry, The University of Tokyo, \\ 7-3-1 Hongo, Bunkyo-ku, Tokyo 113-8656, Japan \\ Koji Horiba and Masaharu Oshima ${ }^{\dagger}$ \\ Department of Applied Chemistry, The University of Tokyo, \\ 7-3-1 Hongo, Bunkyo-ku, Tokyo 113-8656, Japan, and \\ Core Research for Evolutional Science and Technology of Japan Science and Technology Agency, \\ 5 Sanban-cho, Chiyoda-ku, Tokyo 102-0075, Japan, and \\ Synchrotron Radiation Research Organization, The University of Tokyo, \\ 7-3-1 Hongo, Bunkyo-ku, Tokyo 113-8656, Japan \\ Jitsuo Ohta and Hiroshi Fujioka \\ Institute of Industrial Science, The University of Tokyo, \\ 4-6-1 Komaba, Meguro-ku, Tokyo 153-8505, Japan \\ Hisayuki Miki \\ Showa Denko K. K., 5-1 Yawata Kaigan-dori, Ichihara, Chiba 290-0067, Japan
}

Shigenori Ueda, Yoshiyuki Yamashita, Hideki Yoshikawa, and Keisuke Kobayashi

NIMS Beamline Station at SPring-8, National Institute for Materials Science, 1-1-1 Kouto, Sayo, Hyogo 679-5148, Japan

(Received 10 October 2008; Accepted 6 November 2008; Published 22 November 2008)

\begin{abstract}
We have determined the valence band discontinuities of InN/Mg:GaN and InN/Si:GaN heterostructures by hard $\mathrm{x}$-ray photoemission spectroscopy. InN thin films were grown by pulsed laser deposition method and streaky highenergy electron diffraction pattern was observed, indicating that good crystalline InN films were grown. The value of valence band discontinuity for InN/Mg: GaN is as small as $0.3 \mathrm{eV}$, while that for InN/Si:GaN is $1.4 \mathrm{eV}$. The fact that the InN/Mg:GaN interface has the small energy barrier suggests that InN is very promising for the interlayer between transparent conductive layers and $\mathrm{Mg}: \mathrm{GaN}$ in light emitting diode structures.
\end{abstract}

[DOI: $10.1380 /$ ejssnt.2008.254]

Keywords: Synchrotron radiation photoelectron spectroscopy; Epitaxy; Gallium nitride; Indium nitride; Heterojunctions

\section{INTRODUCTION}

After Amano et al. realized $p$-type Mg:GaN [1], groupIII nitride-based semiconductors have attracted much attention because of their direct transition band structures, wide range band gaps and large electron saturation velocities. Among the issues to be solved for realizing the high-brightness light emitting diodes (LEDs), control of the interfaces between metal electrodes and $p$-GaN is very important. Although conventional $\mathrm{Au} / \mathrm{Ni}$ electrodes have very low-resistance ohmic contact to $p$-GaN, its inferior transparency and thermal instability make the device performance insufficient. For the optical improvement, indium tin oxide (ITO) transparent conductive layer is commonly used as an electrode on $p$-GaN because of its good transparency and conductivity. However, as-deposited ITO $/ p$-GaN exhibits very high resistance due to the large Schottky barrier height. Therefore it is necessary to grow the interfacial layer between ITO and $\mathrm{Mg}: \mathrm{GaN}$ in order to reduce the contact resistance $[2-6]$. Since $\mathrm{InN}$ has large electron affinity of $6.1 \mathrm{eV}$ [7] and small band gap of 0.7

* This paper was presented at International Symposium on Surface Science and Nanotechnology (ISSS-5), Waseda University, Japan, 9-13 November, 2008.

†Corresponding author: oshima@sr.t.u-tokyo.ac.jp
$\mathrm{eV}[8]$, it is expected to act as the effective interlayer. In this study, we have investigated both $\mathrm{InN} / \mathrm{Si}: \mathrm{GaN}$ and $\mathrm{InN} / \mathrm{Mg}: \mathrm{GaN}$ interfaces for the determination of valence band discontinuities by high resolution hard x-ray photoemission spectroscopy (HX-PES) in order to examine whether InN is appropriate for the interfacial layer or not.

\section{EXPERIMENTAL}

For the determination of valence band offsets, we have grown three types of samples for photoemission spectroscopy measurements: (i) GaN thick film on a sapphire substrate, (ii) InN thick film on an Yttria-Stabilized Zirconia (YSZ) (111) substrate, and (iii) InN/GaN heterostructure on the sapphire substrate. Each GaN was doped with $\mathrm{Si}$ and $\mathrm{Mg}$ for $n$-type and $p$-type, respectively. (i) and (ii) are used for the determination of valence band maximum (VBM) of GaN and $\mathrm{InN}$ films. (iii) heterostructure samples were grown as follows: $\mathrm{Mg}$-doped $\mathrm{GaN}$ and Si-doped GaN with a thickness of $0.5 \mu \mathrm{m}$ were grown on sapphire substrates by a metalorganic chemical vapor deposition method. The $p$-type Mg-doped GaN film was annealed at $900^{\circ} \mathrm{C}$ in nitrogen ambient to activate the Mg dopant. Next, samples were set into the pulsed laser deposition (PLD) chamber and annealed over $600^{\circ} \mathrm{C}$ for the thermal cleaning of GaN surfaces. InN 
(a) AIN/InN/Si:GaN

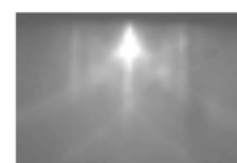

GaN

(b) AIN/InN/Mg:GaN

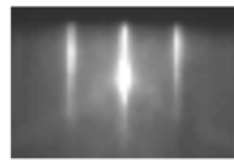

GaN

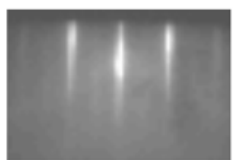

$\ln N$

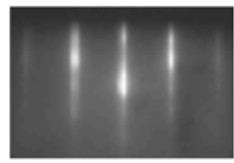

$\ln N$

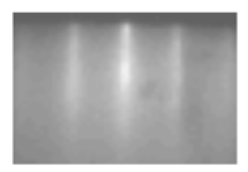

AIN

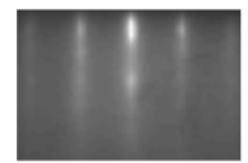

AIN
FIG. 1: RHEED patterns of (a) AlN/InN/Si:GaN and (b) $\mathrm{AlN} / \mathrm{InN} / \mathrm{Mg}: \mathrm{GaN}$.

thin films with a thickness of $\sim 8 \mathrm{~nm}$ were deposited on Si:GaN and Mg:GaN by the PLD method, followed by deposition of AlN which is used as a cap layer to prevent the InN/Mg:GaN interface from oxidization. Sources for InN and AlN were In metal and polycrystalline AlN, respectively. During the growth, nitrogen was supplied through an rf plasma source operated at an input power of $350 \mathrm{~W}$ and at a pressure of $2.0 \times 10^{-5}$ Torr. KrF excimer laser power was kept at $1-10 \mathrm{~mJ} / \mathrm{cm}^{2}$. During the growth, high-energy electron diffraction (RHEED) patterns were observed. Structural information was obtained using transmission electron microscope (TEM) and grazing incidence x-ray reflectivity (GIXR). HX-PES $[9,10]$ was carried out at room temperature under vacuum condition of $\sim 10^{-7} \mathrm{~Pa}$ with the Scienta SES 2002 electron analyzer at SPring-8 BL15XU. For the calibration of the energy scale, Fermi edge of an Au film was used. The total energy resolution was about $300 \mathrm{meV}$ at the photon energy of $6 \mathrm{keV}$. Note that the inelastic mean free path of high-energy excited electrons is estimated to be $\sim 7 \mathrm{~nm}$, which would be appropriate for analyzing buried interfaces.

\section{RESULTS AND DISCUSSION}

Figure 1 shows RHEED patterns for AlN/InN/GaN samples. Sharp streaky patterns in both samples indicate that GaN, InN, AlN were epitaxially grown on sapphire substrates at each growth step. The TEM image of $\mathrm{AlN} / \mathrm{InN} / \mathrm{Mg}: \mathrm{GaN}$ is shown in Fig. 2. It is found that interfacial morphology between InN and $\mathrm{Mg}: \mathrm{GaN}$ is rough. In addition, since surface is fully covered with the thin AlN layer, InN is expected not to be oxidized in air before the ex-situ photoemission measurement. For further information of interfaces, GIXR measurements were performed, as shown in Fig. 3. The AlN/InN/Si:GaN sample shows a typical GIXR oscillation curve, indicating that abrupt interface is formed. Curve fitting analysis using the AlN/InN/Si:GaN structural model reveals that the thicknesses of the AlN and the InN layers are $1.4 \mathrm{~nm}$ and $8.0 \mathrm{~nm}$, and the roughnesses of the AlN surface, the AlN/InN interface, and the InN/Si:GaN inter-

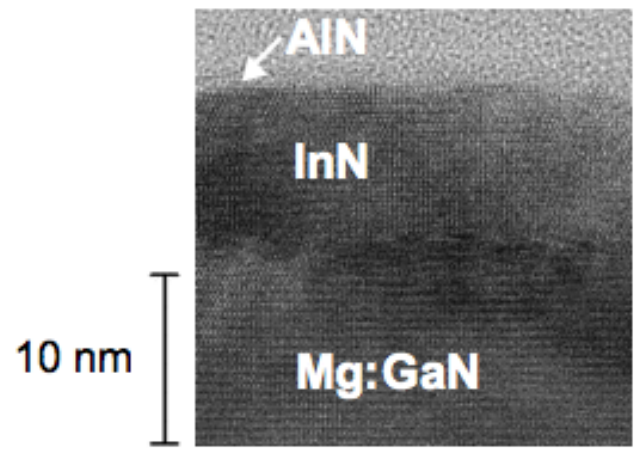

FIG. 2: Cross-sectional TEM image of AlN/InN/Mg:GaN.

(a) AIN/InN/Si:GaN

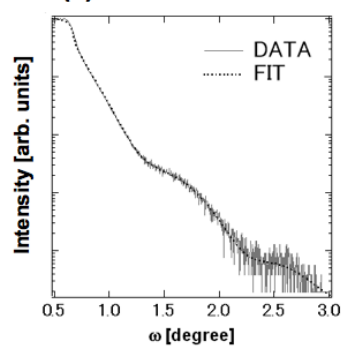

FIG. 3: GIXR patterns of (a) AlN/InN/Si:GaN and (b) AlN/InN/Mg:GaN.

face are $0.58 \mathrm{~nm}, 0.40 \mathrm{~nm}$ and $0.48 \mathrm{~nm}$, respectively. On the other hand, no oscillation curve was observed for the AlN/InN/Mg:GaN sample, which indicates the rough interface. This is consistent with the TEM image. These results suggest that the oxide layer including Mg was not removed by thermal cleaning process, and it brought about the very thin interfacial layer between $\mathrm{InN}$ and $\mathrm{Mg}$ : $\mathrm{GaN}$. Hashizume et al. reported that a disordered layer including $\mathrm{Mg}$ and its oxides is formed at the $p$-type $\mathrm{Mg}$ : $\mathrm{GaN}$ surface [11].

For the epitaxially grown heterojunction samples, the VBM values were determined by HX-PES. At first, we have measured the valence band spectra of thick InN, Si:GaN, and Mg:GaN. Figures 4 (a)-(c) show the HXPES spectra around Fermi energy $\left(E_{\mathrm{F}}\right)$. Valence band spectra were fitted by two straight lines and intersecting points were determined as VBM [12] with the accuracy of of $\pm 0.1 \mathrm{eV}$. For InN, the VBM value was determined to be $1.3 \mathrm{eV}$ and density of states was observed around $E_{\mathrm{F}}$, which indicates the metallic property of the InN. Piper et $a l$. reported that In adlayers and In droplets were formed at the InN surface [13], and Kim et al. measured InN films grown under different growth conditions and insisted that incorporation of oxygen caused the filling of carriers in the conduction band of InN [14]. While the VBM energy of Si:GaN $(3.4 \mathrm{eV})$ is reasonable for $n$-type semiconductor, the VBM value of $\mathrm{Mg}: \mathrm{GaN}$ (2.6 eV) seems high for $p$ type one, considering the fact the band gap of GaN is 3.4 $\mathrm{eV}$ [15]. This may be because the Schottky barrier was generated between the surface oxide layer and $\mathrm{Mg}$ :GaN. However the absolute value does not affect the estimation of valence band discontinuity of $\mathrm{InN} / \mathrm{GaN}$. 
(a) InN valence

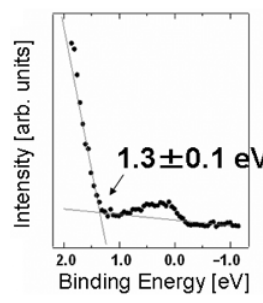

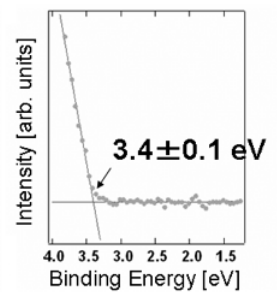

(b) Si:GaN valence (c) Mg:GaN valence

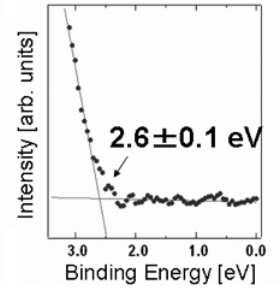

FIG. 4: HX-PES spectra of valence band for (a) InN, (b) $\mathrm{Si}$ GaN and (c) Mg:GaN. (a) $\ln 3 d_{5 / 2}$

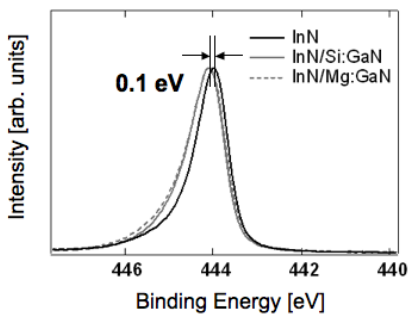

(c)

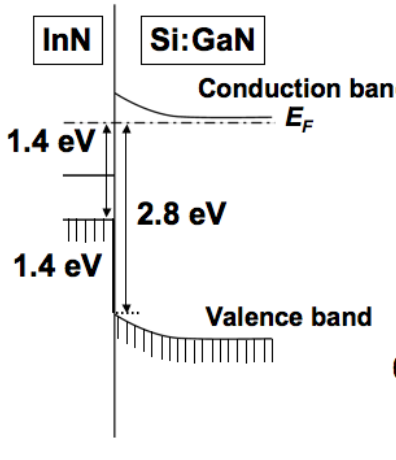

(b) $\mathrm{Ga} 2 p_{3 / 2}$

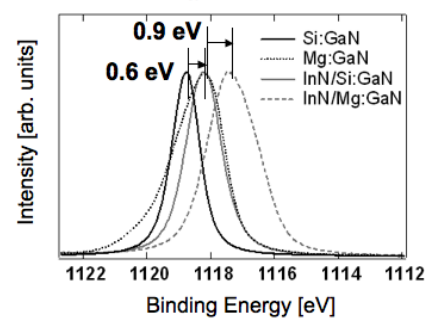

(d)

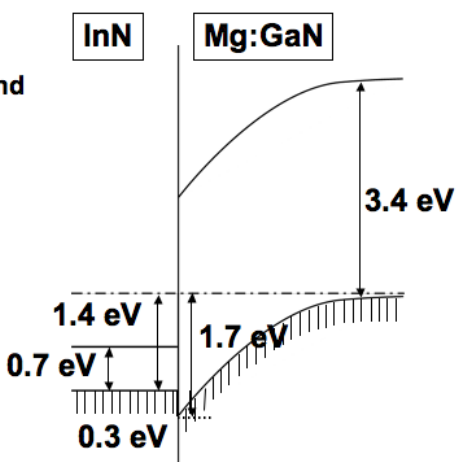

FIG. 5: HX-PES core level spectra for (a) In $3 d_{5 / 2}$ and (b) Ga $2 p_{3 / 2}$, and determined band structures by HX-PES results for (c) InN/Si:GaN and (d) InN/Mg:GaN.

Figures 5 (a) and (b) show core level spectra of $\mathrm{InN} / \mathrm{GaN}$ heterojunction samples. Since core level and VBM should shift by the same value, VBM positions of $\mathrm{InN} / \mathrm{GaN}$ heterojunction samples can be estimated by subtracting the core level shift from the VBM of GaN or InN determined above. Figures 5 (c) and (d) show the experimentally determined band structures of InN/Si:GaN and $\mathrm{InN} / \mathrm{Mg}$ :GaN. Here, $0.7 \mathrm{eV}$ is used as the band gap value of InN. Values of valence band discontinuity are 1.4
$\mathrm{eV}$ and $0.3 \mathrm{eV}$ for InN/Si:GaN and $\mathrm{InN} / \mathrm{Mg}: \mathrm{GaN}$, respectively. These values are different from the reported one of $0.5 \mathrm{eV}$ for $\mathrm{InN} /$ non-doped $\mathrm{GaN}$, which is estimated experimentally [7] and theoretically [16]. This may be because the carrier concentration in $\mathrm{InN}$ was very high and $E_{\mathrm{F}}$ of $\mathrm{Mg}: \mathrm{GaN}$ was pinned by the defects in the oxide layer formed on $\mathrm{Mg}: \mathrm{GaN}$. Although the growth of $\mathrm{InN}$ was done in the vacuum condition and surface was AlNcapped, broad peaks exist in Figs. 5 (a) and (b). This means that $\mathrm{InN}$ and $\mathrm{GaN}$ were oxidized, even for the abrupt InN/Si:GaN probably because of the residual oxide in vacuum chamber. For the InN/Mg:GaN interface, $0.3 \mathrm{eV}$ was achieved probably due to the presence of the Mg-containing oxide layer. Looking into the possibility of low resistance ITO/Mg:GaN interface, InN/Mg:GaN interface having small valence band discontinuity of 0.3 $\mathrm{eV}$ suggests that $\mathrm{InN}$ is very promising for realizing the low resistance ITO/InN/Mg:GaN structure. Note, however, that Fermi level is located at $0.7 \mathrm{eV}$ beyond conduction band minimum (CBM) of InN, indicating that $\mathrm{InN}$ is heavily $n$-type [17], which is similar to the result of thick InN layer on YSZ, as shown in Fig. 4(a). These results suggest that although $n$-type InN interfacial layers in this study cannot be used for $p$-type $\mathrm{Mg}$ : GaN to realize low contact resistivity, a new possibility for the good ohmic contact by the ITO/InN/Mg:GaN structure is shown if high quality $p$-type $\mathrm{InN}$ is successfully grown.

\section{SUMMARY}

Valence band discontinuities were determined by HXPES for InN/Si:GaN and InN/Mg:GaN grown by the PLD method. InN/Mg:GaN interface has the small value of valence band discontinuity $(0.3 \mathrm{eV})$, while $\mathrm{InN} / \mathrm{Si}: \mathrm{GaN}$ interface has the large one $(1.4 \mathrm{eV})$. These values are different from the reported values of $0.5 \mathrm{eV}$, which may be due to the Fermi level pinning by the defects generated in thin oxide layer. Small energy barrier between InN and $\mathrm{Mg}$ : GaN suggests that $\mathrm{InN}$ is very promising for the interlayer of ITO/Mg:GaN ohmic contacts.

\section{Acknowledgments}

The authors would like to thank the staffs of BL15XU, NIMS, and SPring- 8 for their help at the beamline. The authors are also grateful to Dr. M. Arita, Prof. K. Shimada, and Prof. H. Namatame of HiSOR, Hiroshima Univ. for their contribution in the construction of the HXPES experimental station at BL15XU of SPring-8.
[1] H. Amano, M. Kito, K. Hiramatsu, and I. Akasaki, Jpn. J. Appl. Phys. 28, L2112 (1989).

[2] R.-H. Horng, D.-S. Wuu, Y.-C. Lien, and W.-H. Lan, Appl. Phys. Lett. 79, 2925 (2001).

[3] S. Y. Kim, H. W. Jang, and J.-L. Lee, Appl. Phys. Lett. 82, 61 (2003).

[4] K.-M. Chang, J.-Y. Chu, and C.-C. Cheng, Solid-State Electron. 49, 1381 (2005).
[5] J. O. Song, K.-K. Kim, H. Kim, H.-G. Hong, H. Na, and T.-Y. Seong, Electrochem. Solid-State Lett. 10, H270 (2007).

[6] J.-O. Song, K.-K. Kim, H. Kim, Y.-H. Kim, H.-G. Hong, H. Na, and T.-Y. Seong, Mater. Sci. Semicon. Proc. 10, 211 (2007).

[7] C. F. Shih, N. C. Chen, and C. Y. Tseng, Thin Solid Films 516, 5016 (2008). 
[8] J. Wu, W. Walukiewicz, K. M. Yu, J. W. Ager III, E. E. Haller, H. Lu, W. J. Schaff, Y. Saito, and Y. Nanishi, Appl. Phys. Lett. 80, 3967 (2002).

[9] K. Kobayashi, M. Yabashi, Y. Takata, T. Tokushima, S. Shin, K. Tamasaku, D. Miwa, T. Ishikawa, H. Nohira, T. Hattori, Y. Sugita, O. Nakatsuka, A. Sakai, and S. Zaima, Appl. Phys. Lett. 83, 1005 (2003).

[10] K. Kobayashi, Nuclear Instruments and Methods in Physics Research A 547, 98 (2005).

[11] T. Hashizume, J. Appl. Phys. 94, 431 (2003).

[12] S. A. Chambers, T. Droubay, T. C. Kaspar, and M. Gutowski, J. Vac. Sci. Technol. B 22, 2205 (2004).

[13] L. F. J. Piper, T. D. Veal, P. H. Jefferson, C. F. Mc-
Conville, F. Fuchs, J. Furthmuller, F. Bechstedt, Hai Lu, and W. J. Schaff, Phys. Rev. B 72, 245319 (2005).

[14] J. J. Kim, H. Makino, K. Kobayashi, P. P. Chen, E. Ikenaga, M. Kobata, A. Takeuchi, M. Awaji, T. Hanada, M. W. Cho, and T. Yao, Phys. Stat. Sol. (c) 3, 1846 (2006).

[15] H. P. Maruska and J. J. Tietjen, Appl. Phys. Lett. 15, 327 (1969).

[16] S.-H. Wei and A. Zunger, Appl. Phys. Lett. 69, 2719 (1996).

[17] C. G. Van de Walle and J. Neugebauer, Nature 423, 626 (2003). 